\title{
Severe Fever with Thrombocytopenia Syndrome in Patients Suspected of Having Scrub Typhus
}

\author{
Yu Mi Wi, Hye In Woo, Dahee Park, \\ Keun Hwa Lee, Cheol-In Kang, \\ Doo Ryeon Chung, Kyong Ran Peck, \\ Jae-Hoon Song
}

To determine prevalence of severe fever with thrombocytopenia syndrome in South Korea, we examined serum samples from patients with fever and insect bite history in scrub typhus-endemic areas. During the 2013 scrub typhus season, prevalence of this syndrome among patients suspected of having scrub typhus was high $(23.0 \%)$, suggesting possible co-infection.

Severe fever with thrombocytopenia syndrome (SFTS) $\mathcal{N}_{\text {is an emerging infectious disease caused by a novel }}$ phlebovirus in the family Bunyaviridae $(1,2)$. The disease is characterized by fever, gastrointestinal signs and symptoms, leukopenia, and thrombocytopenia $(1,2)$. Exposure to ticks, weeds, and shrubs have been found to be risk factors $(2,3)$.

In South Korea, areas in which SFTS and scrub typhus are endemic overlap $(4,5)$. Scrub typhus is caused by Orientia tsutsugamushi, which are bacteria transmitted to humans by chigger mite bites. Scrub typhus is a major public health problem during harvest season in South Korea (October and November); 10,485 cases were reported in 2013 (5). The clinical presentations of scrub typhus and SFTS are similar; signs and symptoms typically develop within 1-2 weeks of infection and include fever, headache, malaise, and gastrointestinal upset.

In South Korea, febrile patients with a history of bug bites are generally suspected of having scrub typhus and are prescribed antimicrobial drugs (e.g., doxycycline or azithromycin) in the early phases of the disease. We investigated the prevalence of SFTS in patients with fever and a history of insect bites in scrub typhus-endemic areas.

\section{The Study}

During scrub typhus season (September-December) in 2013, we collected serum samples from 74 patients at

Author affiliations: Sungkyunkwan University, Changwon-si,

South Korea (Y.M. Wi, H.I. Woo); Jeju National University School

of Medicine, Jeju-si, South Korea (D. Park, K.H. Lee);

Sungkyunkwan University School of Medicine, Seoul, South Korea

(C-.I. Kang, D.R Chung, K.R. Peck, J.-H. Song)

DOI: http://dx.doi.org/10.3201/eid2211.160597
Samsung Changwon Hospital (Sungkyunkwan University, Changwon-si, South Korea) who had fever and a history of bug bites. At the time of patient admission, we tested for antibodies against $O$. tsutsugamushi by using a commercial immunochromatography kit (SD Bioline Tsutsugamushi Assay; Standard Diagnostics, Yongin, South Korea). For molecular diagnosis of SFTS virus (SFTSV), we performed reverse transcription PCR of partial small RNA segments as previously described (6). We then performed sequencing by using a BigDye Terminator Cycle Sequencing Kit (PerkinElmer Applied Biosystems, Warrington, UK).

Characteristics of SFTSV-positive and -negative populations were compared by using the $\chi^{2}$, Fisher exact, 2 -sample $t$, or Mann-Whitney U tests, as appropriate. Logistic regression was used to identify predictors of SFTS virus infection. Variables for which $p$ value was $<0.05$ in univariate analysis were candidates for multivariate analysis. All analyses were conducted with SPSS for Windows version 18.0 (SPSS Inc., Chicago, IL, USA).

Among the 74 patients who had fever and a history of bug bites during scrub typhus season, the overall prevalence of SFTS infection was $23.0 \%(17 / 74)$. Detected SFTSV sequences showed $97.0 \%-99.0 \%$ identity with the partial sequence of the small RNA segment from SFTSV strains from South Korea (GenBank accession nos. KR612072KR612088). No significant differences were found between the 2 patient groups (with and without SFTS) with regard to farming (Table). Patients infected with SFTSV were much older than those not infected. Among patients infected with SFTSV, clinical presentations of anorexia, nausea/vomiting, and a decreased level of consciousness were more prevalent; lactate dehydrogenase and C-reactive protein levels were remarkably higher; and albumin levels were lower than among patients without SFTSV infection. Incidence of lymphocytopenia was lower among patients with than without SFTSV.

Multivariate analysis revealed that low albumin level at admission (odds ratio $0.19,95 \%$ CI $0.04-0.99 ; \mathrm{p}=$ 0.049 ) and anorexia (odds ratio 13.1, 95\% CI 2.2-78.7; p $=0.005$ ) were independent predictors of SFTS in patients suspected of having scrub typhus. The goodness of fit of the final logistic regression model seemed to be satisfactory (Hosmer-Lemeshow statistic, $\chi^{2}=7.321 ; p=0.396$ ). Of the 17 SFTS patients, 4 had an O. tsutsugamushi antibody titer of 1:2,560, determined by immunochromatography at 
Table. Demographic and laboratory characteristics of SFTS patients, South Korea, 2013*

\begin{tabular}{|c|c|c|c|}
\hline Characteristics & SFTS PCR,$+ n=17$ & SFTS PCR,$- n=57$ & $p$ value \\
\hline Male sex, no (\%) & $7(41.2)$ & $24(42.1)$ & 0.946 \\
\hline Age, mean \pm SD & $64.2 \pm 15.5$ & $54.5 \pm 16.4$ & 0.033 \\
\hline Farming, no (\%) & $12(70.6)$ & $29(50.9)$ & 0.151 \\
\hline \multicolumn{4}{|l|}{ Coexisting condition, no. (\%) } \\
\hline Chronic lung disease† & $3(17.6)$ & $3(5.3)$ & 0.130 \\
\hline Chronic heart disease $\ddagger$ & $6(35.3)$ & $11(19.3)$ & 0.197 \\
\hline Chronic renal disease & $1(5.9)$ & $1(1.8)$ & 0.409 \\
\hline Diabetes & $4(23.5)$ & $5(8.8)$ & 0.197 \\
\hline Chronic liver disease & $1(5.9)$ & $5(8.8)$ & 0.580 \\
\hline Corticosteroid use & 0 & $2(3.5)$ & 0.591 \\
\hline Cancer & 0 & $3(5.3)$ & 0.451 \\
\hline Cerebrovascular disease & $1(5.9)$ & $3(5.3)$ & 0.657 \\
\hline \multicolumn{4}{|l|}{ Clinical presentation } \\
\hline Fever (temperature $\geq 38.3^{\circ} \mathrm{C}$ ) & $13(76.5)$ & $48(84.2)$ & 0.480 \\
\hline Headache & $5(29.4)$ & $18(31.6)$ & 0.865 \\
\hline Myalgia & $8(47.1)$ & $31(54.4)$ & 0.595 \\
\hline Anorexia & $10(58.8)$ & $5(8.8)$ & $<0.001$ \\
\hline Nausea/vomiting & $8(47.1)$ & $10(17.5)$ & 0.022 \\
\hline Abdominal pain & $2(11.8)$ & $5(8.8)$ & 0.657 \\
\hline Diarrhea & $1(5.9)$ & $1(1.8)$ & 0.409 \\
\hline Cough & $1(5.9)$ & $5(8.8)$ & 0.580 \\
\hline Dyspnea & $1(5.9)$ & $2(3.5)$ & 0.549 \\
\hline Decreased consciousness & $3(17.6)$ & - & 0.010 \\
\hline Rash & $12(70.6)$ & $34(59.6)$ & 0.414 \\
\hline \multicolumn{4}{|l|}{ Laboratory findings at admission } \\
\hline Leukopenia $\left(<4,000\right.$ cells $\left./ \mathrm{mm}^{3}\right)$ no. $(\%)$ & $2(11.8)$ & $18(31.6)$ & 0.131 \\
\hline Lymphocytopenia $\left(<1,500\right.$ cells $\left./ \mathrm{mm}^{3}\right)$ no. $(\%)$ & $6(35.5)$ & $43(75.4)$ & 0.002 \\
\hline Anemia (hematocrit <30\%), no. (\%) & $3(17.6)$ & $4(7.0)$ & 0.341 \\
\hline Thrombocytopenia $\left(<10^{6} \mathrm{cells} / \mathrm{mm}^{3}\right)$ no. $(\%)$ & $7(41.2)$ & $13(22.8)$ & 0.221 \\
\hline CPK, IU/L, median (IQR) & $67(33-132)$ & $76(45-128)$ & 0.512 \\
\hline $\mathrm{LDH}, \mathrm{IU} / \mathrm{L}$, mean $\pm \mathrm{SD}$ & $533 \pm 202$ & $402 \pm 151$ & 0.021 \\
\hline AST, IU/L, median (IQR) & $104(48-194)$ & $69(54-112)$ & 0.210 \\
\hline ALT, IU/L, median (IQR) & $70(30-119)$ & $53(35-83)$ & 0.616 \\
\hline PT (INR), median (IQR) & $1.08(1.03-1.15)$ & $1.03(0.97-1.09)$ & 0.057 \\
\hline CRP, mg/L, median (IQR) & $71.6(46.4-110.4)$ & $42.9(23.2-80.3)$ & 0.034 \\
\hline BUN, mg/dL, median (IQR) & $12.7(9.3-18.2)$ & $12.7(9.4-15.3)$ & 0.634 \\
\hline Creatinine, $\mathrm{mg} / \mathrm{dL}$, median (IQR) & $0.9(0.8-1.3)$ & $0.8(0.6-1.0)$ & 0.510 \\
\hline Albumin, $\mathrm{g} / \mathrm{dL}$, mean $\pm \mathrm{SD}$ & $2.9 \pm 0.7$ & $3.3 \pm 0.5$ & 0.005 \\
\hline Hematuria, no. (\%) & $3(20.0)$ & $20(35.7)$ & 0.356 \\
\hline \multicolumn{4}{|l|}{ Outcome } \\
\hline Intensive respiratory or vasopressor support & $3(17.6)$ & $1(1.8)$ & 0.036 \\
\hline $\begin{array}{l}\text { Time from symptom onset to admission, } d \text {, } \\
\text { median (IQR) }\end{array}$ & $3(3-6.5)$ & $4(3-6)$ & 0.599 \\
\hline Hospital stay, d, median (IQR) & $0(0-3)$ & $1(0-4)$ & 0.432 \\
\hline Death, no (\%) & $1(5.9)$ & 0 & 0.230 \\
\hline \multicolumn{4}{|c|}{$\begin{array}{l}{ }^{*} \mathrm{ALT} \text {, alanine aminotransferase; AST, aspartate aminotransferase; BUN, blood urea nitrogen; CPK, creatinine phosphokinase; CRP, C-reactive } \\
\text { protein; INR, international normalized ratio; IQR, interquartile range; LDH, lactate dehydrogenase; PT, prothrombin time; SFTS, severe fever with } \\
\text { thrombocytopenia syndrome. } \\
\text { †Asthma, chronic obstructive pulmonary disease, bronchiectasis. } \\
\text { †Underlying coronary heart disease, chronic heart failure. }\end{array}$} \\
\hline
\end{tabular}

admission, and 3 showed seroconversion on paired serum samples. Those infected with SFTSV were more likely to require intensive respiratory or vasopressor support than those not infected. During hospitalization, 1 (5.9\%) SFTS patient died. Length of hospital stay was similar between patients infected and not infected with SFTSV.

\section{Conclusions}

In scrub typhus-endemic areas of South Korea, prevalence of SFTS among patients with suspected scrub typhus is quite high (23.0\%). Independent predictors of SFTS in patients with suspected scrub typhus are low albumin level at admission and anorexia. Co-infection with SFTSV and $O$. tsutsugamushi was suspected for 7 patients. Patients with SFTS experienced a more severe clinical illness; however, outcomes such as death and length of hospital stay did not vary significantly between groups of patients with and without SFTSV.

As an emerging infectious disease, SFTS is an increasing public health threat because of its wide distribution 
and high mortality rate $(1,2)$. In South Korea during April-December 2013, a total of 35 cases of SFTS were reported (4). The major signs and symptoms of these 35 patients were fever $(100 \%)$, gastrointestinal upset (74\%), fatigue $(74 \%)$, thrombocytopenia $(100 \%)$, and leukocytopenia (100\%) (4). During 2013, the reported mortality rate among patients with SFTS in South Korea was 45.7\% (16 deaths/35 patients), which is higher than that reported in China $(6 \%-30 \%)(1,2)$. However, our study found a mortality rate of $5.9 \%$, and illness was severe in only $3(17.6 \%)$ patients. A large-scale serologic survey of 2,547 farmers living in rural areas of Jiangsu Province in China (7) found an overall SFTSV antibody prevalence rate of $1.30 \%$, and the farmers seropositive for SFTS did not report having typical symptoms of SFTS. This finding suggests occurrence of asymptomatic or mild cases of SFTS.

In our study, all SFTS patients initially received a diagnosis of scrub typhus because of its seasonality and the similar presentation of the 2 diseases. The Korea Center for Disease Control and Prevention and the National Notifiable Disease Surveillance System reported that during 2013, SFTS occurred mainly from April to September (4). However, we showed that SFTS was also prevalent in October and November (Figure).

SFTS is mainly transmitted to humans by SFTSVinfected ticks; however, person-to-person transmission by direct contact with infected blood or body fluids has also been reported $(8,9)$. Therefore, standard precautions are necessary for healthcare workers in contact with patients with fever and a history of bug bites.

Previous studies have suggested that Haemaphysalis longicornis and Rhipicephalus microplus ticks are the most likely vectors of SFTSV transmission to humans $(10,11)$. $H$. longicornis ticks are widespread in South Korea and their

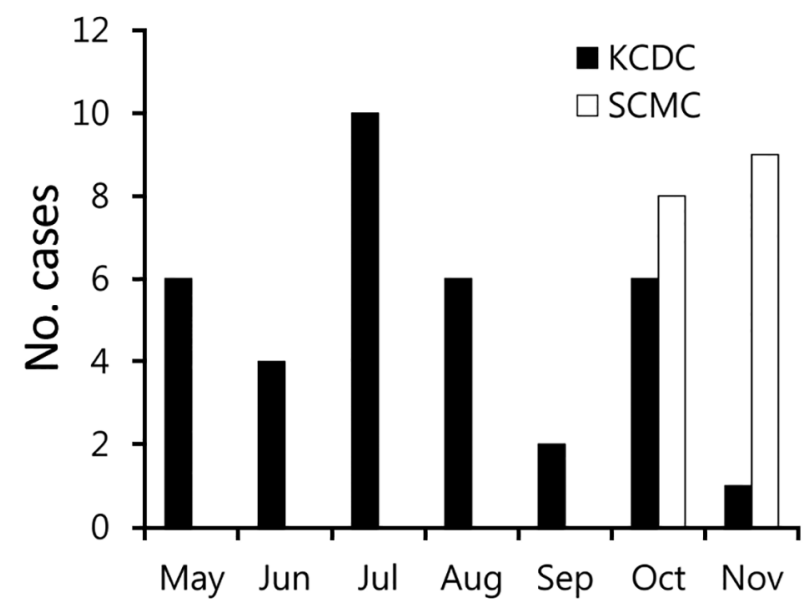

Figure. Seasonal distribution of severe fever with thrombocytopenia syndrome, South Korea, 2013. KCDC, Korea Center for Disease Control and Prevention; SCMC, Samsung Changwon Hospital. density is high during May-August, when temperatures are usually warm (10). In contrast, Leptotrombidium scutellare mites are major scrub typhus vectors with high density during autumn (scrub typhus season) (12). Therefore, for patients with fever and a history of bug bites, physicians in South Korea tend to suspect SFTS during summer and scrub typhus during autumn. In our study, the antibody titer of 1:2,560 in 7 patients suggests the possibility of SFTSV and $O$. tsutsugamushi co-infection. In China, SFTSV has also been detected by reverse transcription PCR in $L$. scutellare mites (13). Therefore, further research is needed to confirm the mite-SFTSV association in addition to the prevalence of SFTSV and $O$. tsutsugamushi co-infection.

The results of this study suggest that in South Korea, prevalence of SFTS is quite high among patients suspected of having scrub typhus. Signs and symptoms of SFTS can be atypical. Therefore, healthcare workers in contact with patients suspected of having scrub typhus should take standard precautions. Further epidemiologic research is needed to improve ability to accurately differentiate SFTS from other diseases and to confirm the vector of SFTS.

This study was supported by a Samsung Biomedical Research Institute grant.

Dr. Wi is an assistant professor in the Divisions of Infectious Diseases at Sungkyunkwan University, Changwon-si, South Korea. Her research interest is the epidemiology of tickborne infectious diseases.

\section{References}

1. Yu XJ, Liang MF, Zhang SY, Liu Y, Li JD, Sun YL, et al. Fever with thrombocytopenia associated with a novel bunyavirus in China. N Engl J Med. 2011;364:1523-32. http://dx.doi.org/10.1056/ NEJMoa1010095

2. Liu Q, He B, Huang SY, Wei F, Zhu XQ. Severe fever with thrombocytopenia syndrome, an emerging tick-borne zoonosis. Lancet Infect Dis. 2014;14:763-72. http://dx.doi.org/10.1016/ S1473-3099(14)70718-2

3. Du Z, Wang Z, Liu Y, Wang H, Xue F, Liu Y. Ecological niche modeling for predicting the potential risk areas of severe fever with thrombocytopenia syndrome. Int J Infect Dis. 2014;26:1-8. http://dx.doi.org/10.1016/j.ijid.2014.04.006

4. Park SW, Han MG, Yun SM, Park C, Lee WJ, Ryou J. Severe fever with thrombocytopenia syndrome virus, South Korea, 2013. Emerg Infect Dis. 2014;20:1880-2. http://dx.doi.org/10.3201/ eid2011.140888

5. Lee HW, Cho PY, Moon SU, Na BK, Kang YJ, Sohn Y, et al. Current situation of scrub typhus in South Korea from 2001-2013. Parasit Vectors. 2015;8:238. http://dx.doi.org/10.1186/s13071-015-0858-6

6. Yun Y, Heo ST, Kim G, Hewson R, Kim H, Park D, et al. Phylogenetic analysis of severe fever with thrombocytopenia syndrome virus in South Korea and migratory bird routes between China, South Korea, and Japan. Am J Trop Med Hyg. 2015;93:468-74. http://dx.doi.org/10.4269/ajtmh.15-0047

7. Li Z, Hu J, Bao C, Li P, Qi X, Qin Y, et al. Seroprevalence of antibodies against SFTS virus infection in farmers and animals, Jiangsu, China. J Clin Virol. 20141;60:185-9. http://dx.doi.org/10.1016/j.jcv.2014.03.020 
8. Kim WY, Choi W, Park SW, Wang EB, Lee WJ, Jee Y, et al. Nosocomial transmission of severe fever with thrombocytopenia syndrome in Korea. Clin Infect Dis. 2015;60:1681-3. http://dx.doi.org/10.1093/cid/civ128

9. Gai Z, Liang M, Zhang Y, Zhang S, Jin C, Wang SW, et al. Person-to-person transmission of severe fever with thrombocytopenia syndrome bunyavirus through blood contact. Clin Infect Dis. 2012;54:249-52. http://dx.doi.org/10.1093/cid/ cir776

10. Yun SM, Lee WG, Ryou J, Yang SC, Park SW, Roh JY, et al. Severe fever with thrombocytopenia syndrome virus in ticks collected from humans, South Korea, 2013. Emerg Infect Dis. 2014;20:1358-61. http://dx.doi.org/10.3201/eid2008.131857

11. Luo LM, Zhao L, Wen HL, Zhang ZT, Liu JW, Fang LZ, et al. Haemaphysalis longicornis ticks as reservoir and vector of severe fever with thrombocytopenia syndrome virus in China. Emerg Infect Dis. 2015;21:1770-6. http://dx.doi.org/10.3201/ eid2110.150126
12. Roh JY, Song BG, Park WI, Shin EH, Park C, Park MY, et al. Coincidence between geographical distribution of Leptotrombidium scutellare and scrub typhus incidence in South Korea. PLoS One. 2014;9:e113193. http://dx.doi.org/10.1371/journal.pone.0113193

13. Wang QK, Ge HM, Li ZF, Shan YF, Cui L, Wang YP. Vector research of severe fever with thrombocytopenia syndrome virus in gamasid mites and chigger mites [in Chinese]. Chinese Journal of Vector Biology and Control. 2012;23:452-4.

Address for correspondence: Kyong Ran Peck, Division of Infectious Diseases, Samsung Medical Center, Sungkyunkwan University School of Medicine, 50 Irwon-dong, Gangnam-gu, Seoul 135-710, Republic of Korea; email: krpeck@skku.edu; Keun Hwa Lee, Department of Microbiology and Immunology, Jeju National University School of Medicine, 15, Aran 13 gil, Jeju-si, Jeju Special-Governing Province, 690-756, Republic of Korea; email: yomust7@jejunu.ac.kr

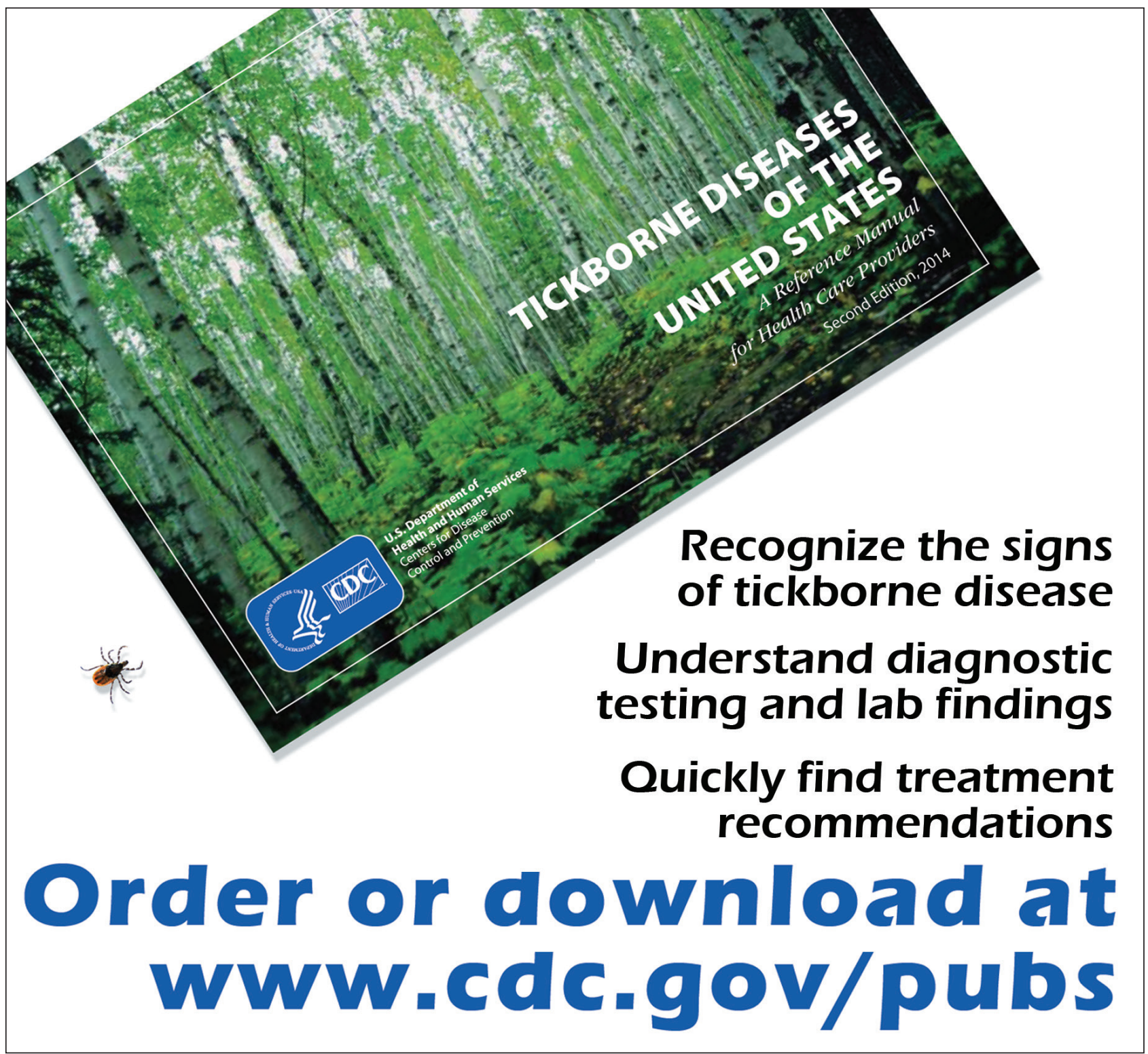

